\title{
fitude \\ des tourbillons de Taylor dans des espaces très petits : Visualisation, mesure du couple et anémométrie
}

\author{
par' J. Frêne \\ Maitre-Assistant, \\ Laboratoire de Mécanique des Contazts, \\ Villeurbanne
}

\section{Introduction}

On sait, à la suite des travaux de Taylor [1], que sous certaines conditions, des tourbillons, dits tourbillons de Taylor, se produisent dans l'espace annulaire défini par deux cylindres concentriques en rotation. L'apparition de ces tourbillons s'accompagne naturellement d'une élévation importante des pertes par frottement visqueux. Ce phénomène, confirmé expérimentalement dans des conditions que nous préciserons plus tard, peut se produire dans le film lubrifiant des paliers des groupes turbo-alternateurs de grande dimension, ou dans les paliers des machines tournantes utilisant des fluides à faible viscosité comme lubrifiant [2], par exemple les paliers Iubrifiés au sodium des pompes des réacteurs nucléaires de type Phénix.

Du point de vue théorique, Taylor a étudié le cas des cylindres coaxiaux, or l'arbre d'un palier en fonctionnement est excentré par rapport au coussinet. Du point de vue expérimental, les résultats déjà publiés ont été obtenus avec des jeux radiaux très supérieurs à ce que l'on trouve en technologie.

Nous nous proposons dans le cadre de cette étude :

- de faire un bref rappel, sur la théorie de Taylor, sur ses prolongements récents qui tiennent compte de l'excentration des cylindres et sur les résultats expérimentaux publiés dans la littérature;

- de donner des résultats originaux obtenus avec des jeux radiaux représentatifs de ceux qui existent dans les paliers.

\section{La théorie \\ de Taylor et ses prolongements modernes}

En 1923, Taylor a montré [1], en utilisant la méthode des perturbations que des instabilités tourbillonnaires (figure 1) apparaissaient entre deux cylindres concentriques quand un nombre sans dimension $P$ donné par l'expression :

$$
F=\frac{\pi^{4} \nu^{2}\left(R_{1}+R_{2}\right)}{2 \Omega^{2}{ }_{1} C^{3} R^{2}{ }_{1}\left[1-m\left(R^{2}{ }_{2} / R^{2}{ }_{1}\right)\right](1-m)}
$$

prenait une valeur critique. Dans le cas où $C / R_{1}$ est petit mais non négligeable, cette valeur est donnée par la relation:

$$
\begin{aligned}
& P_{c}=0,0571\left(\frac{1+m}{1-m}-0,652 \frac{C}{R_{1}}\right) \\
& \quad+0,00056\left(\frac{1+m}{1-m}-0,652 \frac{C}{R_{1}}\right)^{-1}
\end{aligned}
$$

Dans ces expressions, $\nu$ est la viscosité cinématique du fluide, $R_{1}$ et $R_{2}$ respectivement les rayons des cylindres intérieurs et extérieurs animés des vitesses angulaires $\Omega_{1}$ et $\Omega_{2}, C=R_{2}-R_{1}$ le jeu radial et $m=\Omega_{2} / \Omega_{1}$ le rapport des vitesses angulaires.

Dans le cas des paliers lisses, pour lesquels $\Omega_{\mathrm{g}}$ et $m$ sont nuls et où le jeu radial $C$ est très petit, on définit un nombre de Taylor proportionnel à l'inverse de la racine carrée de $P$ par la relation:

$$
T=\frac{\Omega R C}{\nu} \sqrt{\frac{C}{R}}=\mathscr{R e} \sqrt{\frac{C}{R}}
$$

dont la valeur critique est 41,2 . 


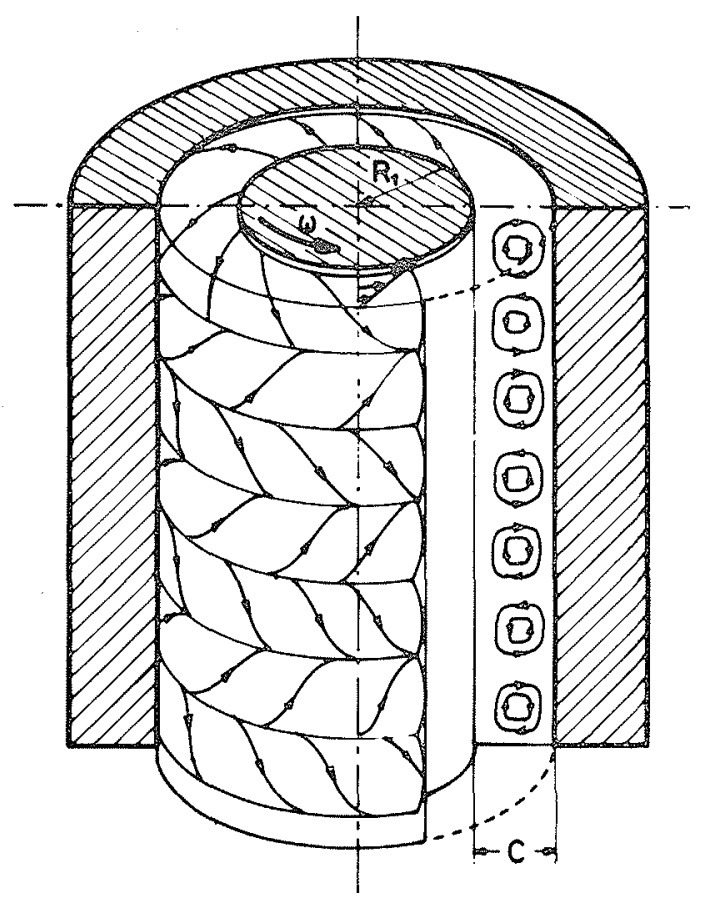

1/ Représentation des tourbillons de Taylor [21].

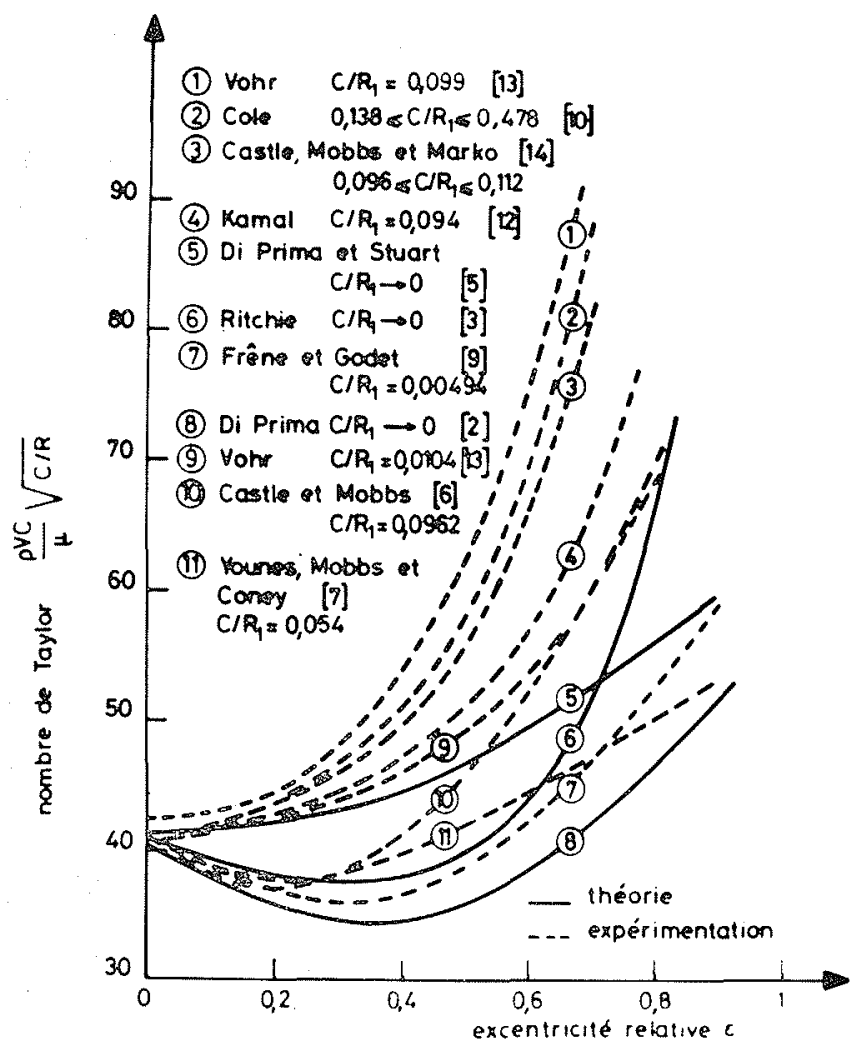

2/ Courbes de variation du nombre de Taylor critique avec l'excentricité relative.
Entre 1963 et 1972, Di Prima [3], Ritchie [4] et Urban et Krueger [5] ont montré indépendamment que, dans le cas de cylindres excentrés, la valeur du nombre de Taylor critique n'est plus constante, mais décroît d'abord pour croître ensuite avec l'excentricité (figure 2). Dans cette première série d'études, les auteurs utilisent la notion de stabilité locale qui suppose l'écoulement localement parallèle. Ils montrent également que les tourbillons apparaissent d'abord là où le jeu est maximal. Très récemment, Di Prima et Stuart [6] ont publié une théorie plus générale qui tient compte de la composante radiale de l'écoulement dans le fluide; ils montrent que la valeur du nombre de Taylor critique crôt avec l'excentricité relative (figure 2). Par ailleurs, la zone de plus forte intensité tourbillonnaire serait située $90^{\circ}$ en aval du point où le jeu radial est maximal. Précisons que toutes ces études ont été effectuées en supposant la longueur des cylindres infinie et le jeu radial très faible.

\section{Les données expérimentales existantes}

Du point de vue expérimental, les résultats présentent une certaine dispersion (figure 2) et les conclusions sont différentes d'un auteur à l'autre: d'une part Castle et Mobbs [7], Younes, Mobbs et Coney [8] et Frêne et Godet $[9,10]$ obtiennent un nombre de Taylor critique qui décroît d'abord puis croît ensuite lorsque l'excentricité augmente; d'autre part Cole [11, 12] Kamal [13], Vohr [14] et Castle, Mobbs et Marko [15] montrent que le nombre de Taylor critique croît avec l'excentricité relative. Par ailleurs, Vohr note, dans un cas particulier, que les tourbillons sont le plus développés approximativement à $50^{\circ}$ en aval du point d'épaisseur maximale; il constate d'autre part que, pour une même excentricité, le nombre de Taylor critique décroît très sensiblement lorsque le rapport $C / R$ décroît; alors que Cole note que le nombre de Taylor critique est indépendant de ce rapport $C / R$.

On voit que les résultats expérimentaux existant ne permettent pas de choisir entre les théories locales et la théorie plus générale de Di Prima et Stuart.

\section{Programme expérimental}

Plan des expériences

Dans une première partie, des essais de visualisation de l'écoulement et de mesure du couple permettront, dans le cas de cylindres coaxiaux, de vérifier ou d'infirmer l'existence de tourbillons de Taylor dans des espaces très réduits. Dans une seconde partie, les informations obtenues, dans le cas de cylindres coaxiaux, à l'aide d'un anémomètre ou sonde à film chaud, seront comparées à celles obtenues ci-dessus. Dans une troisième partie, l'étude de la structure de l'écoulement entre deux cylindres excentrés sera abordée. Le dispositif d'essai ne permettant pas d'imposer une excentricité donnée, les mesures seront effectuées, dans le cas de cylindres excentrés, sous l'influence d'une instabilité mécanique bien connue dans les paliers lisses sous le nom de «Whirl» ou fouettement $\left.{ }_{\alpha} 16\right]$. Cette instabilité impose 
au cylindre extérieur un mouvement radial d'amplitude sensiblement constante et de vitesse angulaire légèrement inférieure à la moitié de la vitesse angulaire du cylindre intérieur en rotation; ainsi, les points d'épaisseur minimale et maximale intéressent successivement toutes les génératrices du cylindre extérieur et, en particulier, celle qui porte la sonde pariétale.

\section{Le disposilif expérimental}

Le dispositif d'essai et l'ensemble du système de mesure est présenté figure 3. La géométrie de ce dispositif est celle d'un viscosimètre de Couette [17]. Le cylindre intérieur, en acier de rayon $R=40,97 \mathrm{~mm}$ et de longueur $L=90 \mathrm{~mm}$, est entrainé en rotation à vitesse variable entre 950 et $3000 \mathrm{tr} / \mathrm{mn}$. Cette vitesse est mesurée avec une précision supérieure à $0,5 \%$ à l'aide d'une roue dentée, d'un capteur magnétique et d'un compteur d'impulsions. Une butée à air supporte le cylindre extérieur pour permettre la mesure du couple d'entraînement. Un couplemètre à jauge d'extensométrie, monté sous la butée à air dans l'axe de rotation de la machine, permet de mesurer des couples variables entre 0,002 et $2 \mathrm{mN}$. Un thermocouple placé juste au-dessus du cylindre intérieur donne la température du fluide avec une précision de $0,5^{\circ} \mathrm{C}$, le fluide utilisé est de l'eau distillée. Le cylindre extérieur est soit en verre rectifié et poli, soit en bronze. Pour les essais de visualisation, de fines particules d'aluminium sont en suspension dans l'eau distillée. Des mesures ont montré que la présence de ces particules ne modifie pas la viscosité et la masse volumique du fuide.

Les mesures anémométriques nécessitent un équipement complémentaire $[18,19]$. Dans la paroi du cylindre extérieu: est fixée une sonde à film chaud (DISA 55 A 93) dont le film de $0,75 \mathrm{~mm}$ de longueur et $0,15 \mathrm{~mm}$ de largeur, affleure à la paroi et est perpendiculaire à la direction de l'écoulement. Cette sonde est alimentée par un anémomètre à température constante (DISA $55 \mathrm{M} \mathrm{10)}$ ). La tension $U$ de sortie de l'anémomètre, qui est liée à la contrainte $\tau$ de cisaillement à la paroi par une relation de la forme $U^{2}=A \tau^{1 / 3}+B$, où $A$ et $B$ sont des constantes d'étalonnage, s'écrit d'une façon classique $U=\bar{U}+u^{\prime}$, où $\bar{U}$ est la valeur moyenne et $u^{\prime}$ la fluctuation. La composante continue $\bar{U}$ est mesurée directement sur un voltmètre; la composante alternative $u^{\prime}$ est dirigée d'une part sur un oscilloscope multivoie, d'autre part sur un voltmètre efficace qui donne directement la racine carrée, Vrms, de la moyenne du carré de $u^{\prime}$ :

$$
V r m s=\sqrt{\frac{1}{T} \int_{t}^{t+T^{\prime}} u^{\prime 2} d t}
$$

Le temps d'intégration $T$ est, dans nos essais, très supérieur à la période de rotation du cylindre intérieur. Par ailleurs, sur la génératrice du cylindre extérieur où est située la sonde à film chaud, est fixé un capteur de mesure de positionnement sans contact (Vibrometer Tw 5) qui permet de mesurer l'épaisseur du film au droit de la sonde; ce capteur est alimenté par un pont à fréquence porteuse de $8 \mathrm{kHz}$ dont la tension de sortie est fonction de l'épaisseur du film en regard du capteur; le signal ainsi obtenu est dirigé sur une des voies de l'oscilloscope. La position angulaire du cylindre intérieur est aussi mesurée par un capteur magnétique qui donne un top tous les $36^{\circ}$; ce signal est dirigé sur une troisième vole de l'oscilloscope.

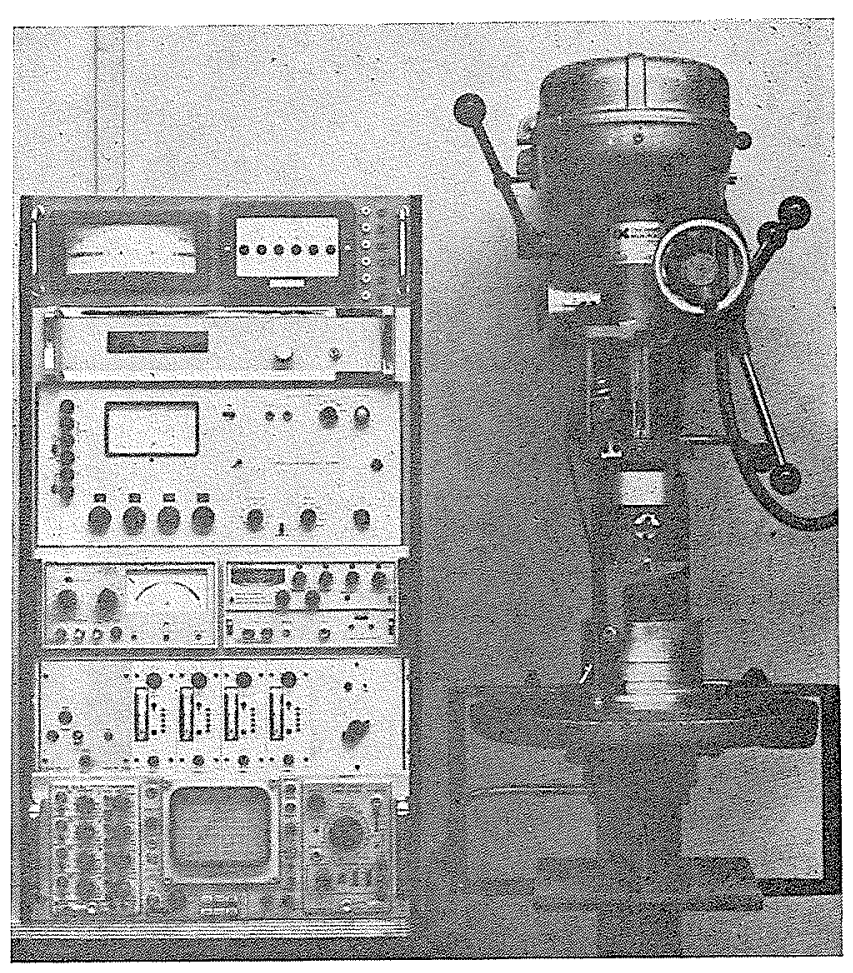

3/ Vue d'ensemble de la machine et des appareils de mesure.

\section{Résultats}

Résultats obtenus par visualisation et par mesure du
couple

Les essais de visualisation ont été effectués avec deux cylindres extérieurs donnant respectivement des jeux radiaux de $0,225 \mathrm{~mm}$ et $0,127 \mathrm{~mm}$, soit des rapports $C / R$ de 0,0055 et 0,0031 . Les figures 4 à 9 sont des photographies de l'écoulement obtenu à différentes vitesses pour un $C / R$ de 0,0055 et la figure 10 pour un $C / R$ de 0,0031 ; l'agrandissement de cette dernière figure est différent de celui des autres. Chaque petite division au bas des photos représente un millimètre. Par ailleurs, chacun des tores visibles sur es photos est composé de deux tourbillons tournant en sens inverse l'un de l'autre (figure 1).

Les figures 4 et 5 montrent, qu'en accord avec la théorie, les tourbillons se développent dans le fluide, même dans le cas de jeux très petits. Lorsque la vitesse augmente (figure 6 et 7) les tourbillons ne restent pas totalement parallèles entre eux, cependant il n'apparaît pas, comme dans le cas de jeux plus importants [20], une périodicité très nette dans la direction circonférentielle. A grande vitesse, malgré l'apparition (figure 8) et le développement (figure 9) de la turbulence, la structure des tourbillons de Taylor reste encore apparente. Les essais effectués pour un rapport $C / R$ de 0,0031 permettent d'observer les mêmes phénomènes (figure 10). 


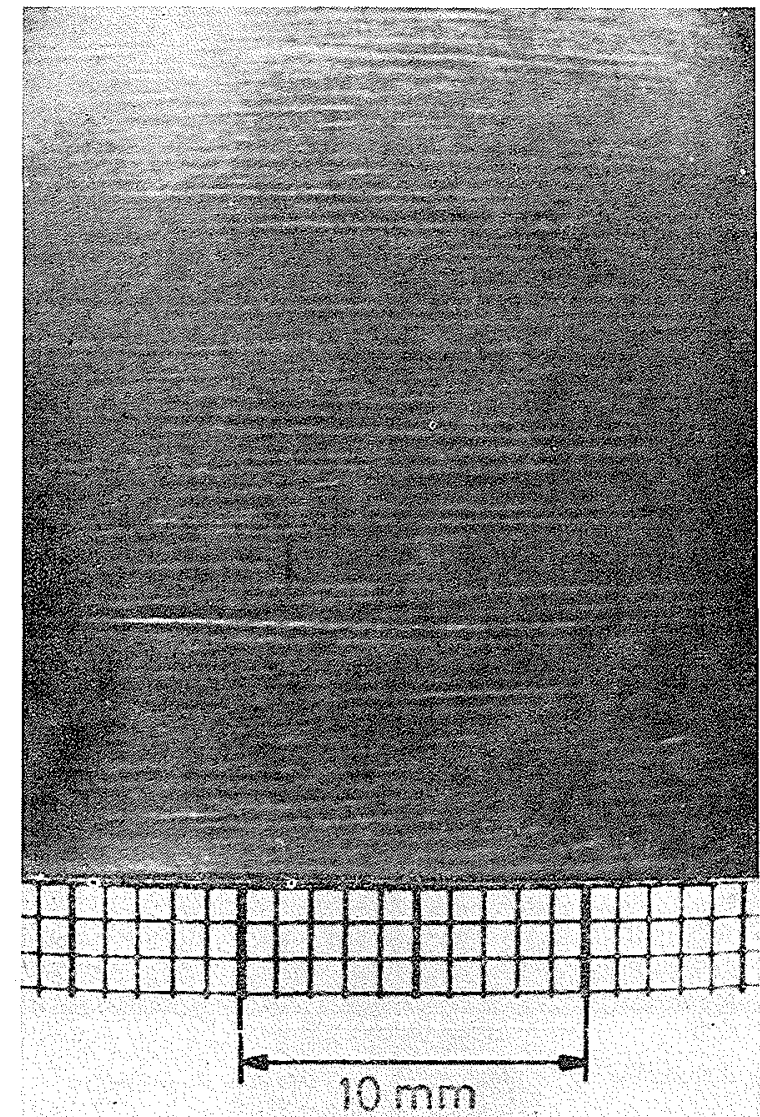

14

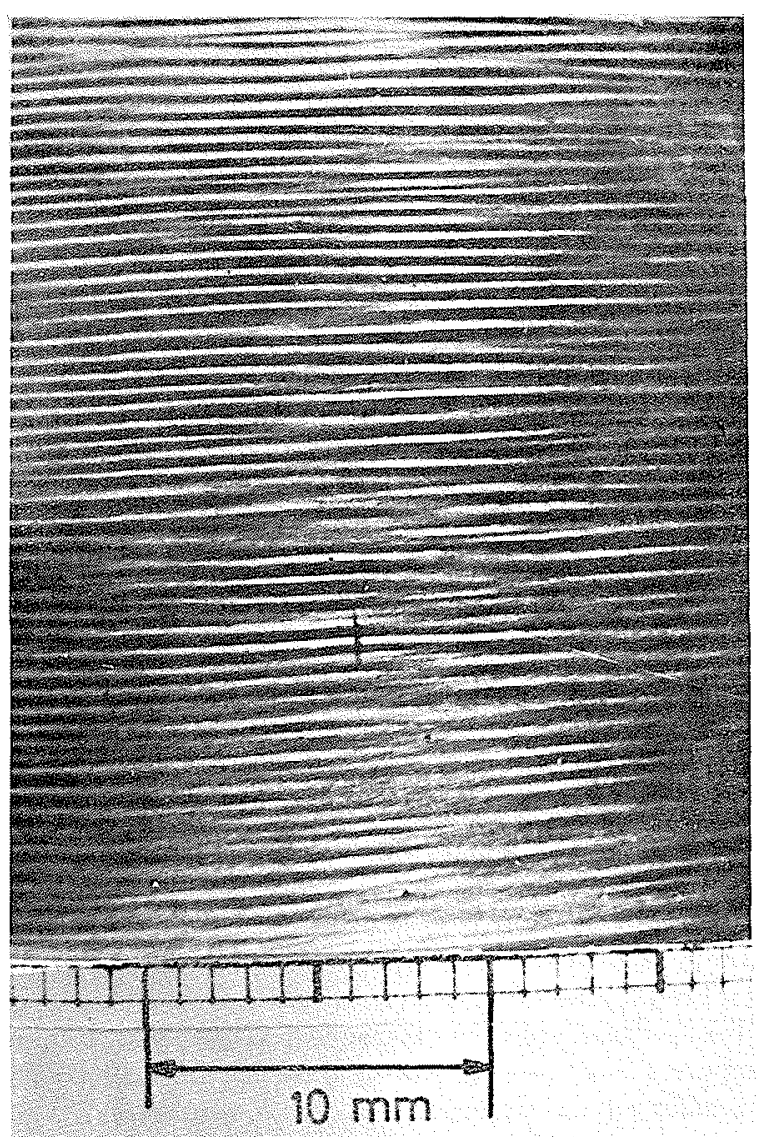

6)

$10 \mathrm{~mm}$

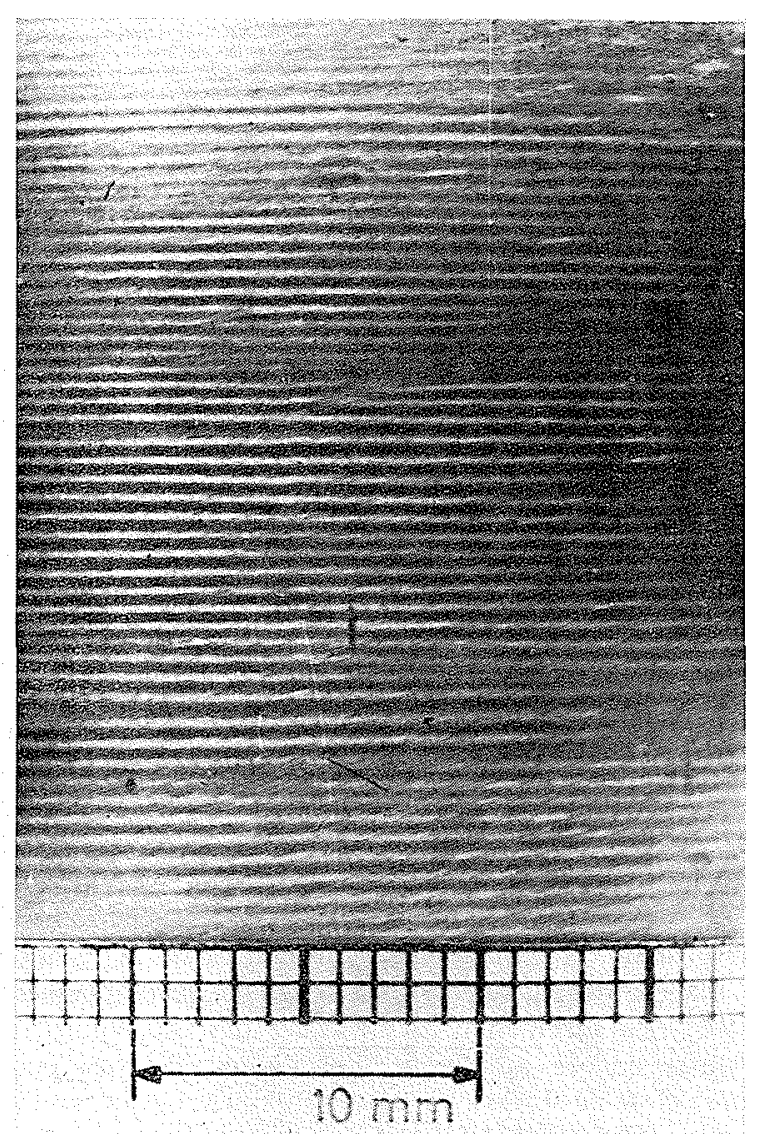

15

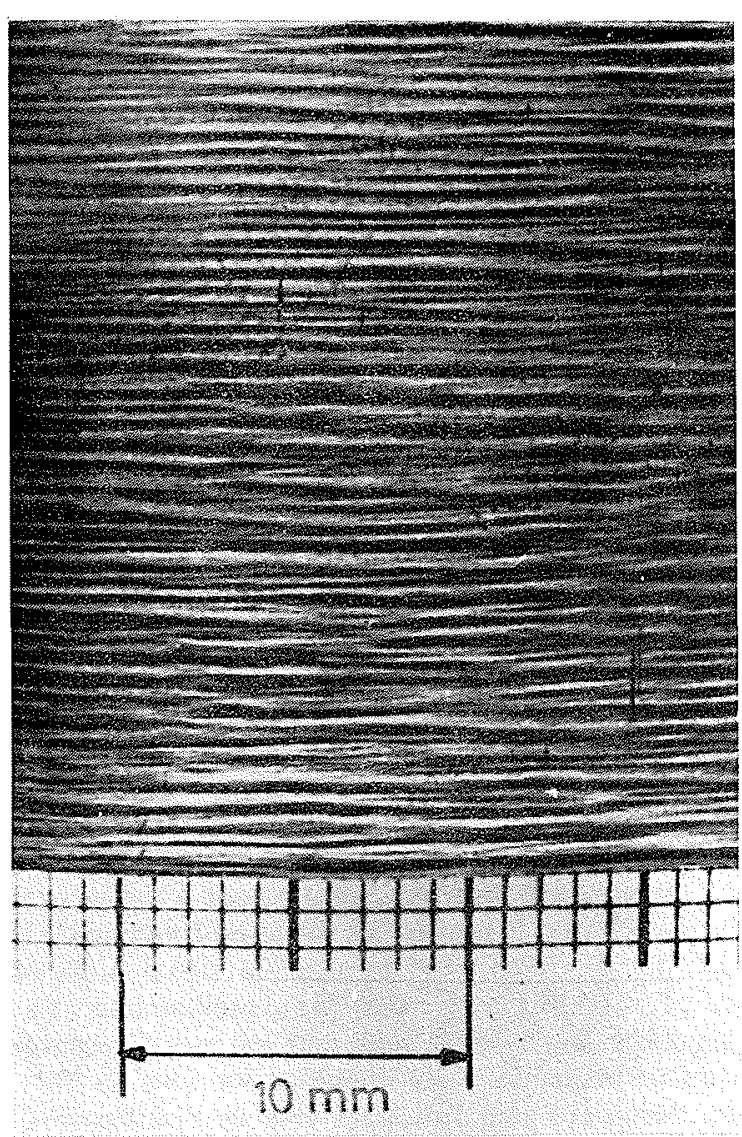




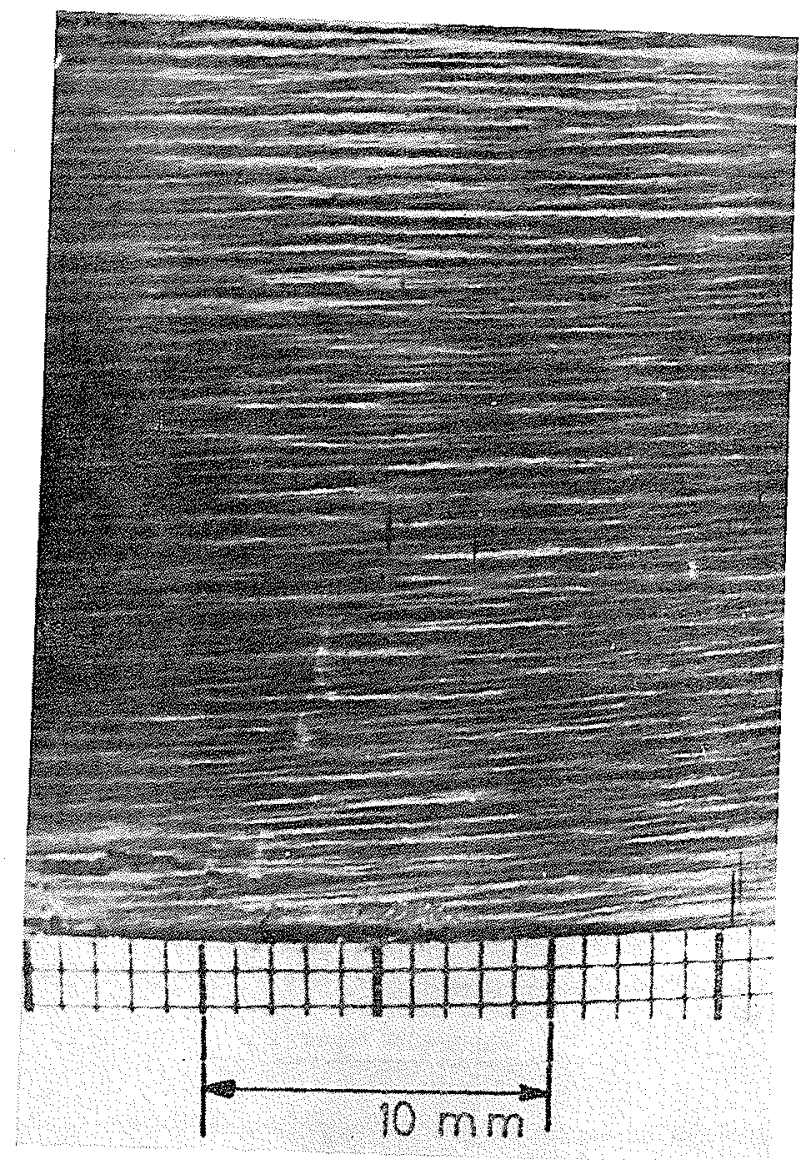

8)

4/ Naissance des premiers tourbillons.

$\mathrm{T}=40,4-R \mathrm{c}=545-N=442 \mathrm{tr} / \mathrm{mn}$

5) Tourbillons de Taylor juste après la transition. $T=42,9-R \mathrm{e}=578-N=470 \mathrm{tr} / \mathrm{mn}$.

6) Apparition des premlères distorsions. $T=54,9-\operatorname{Re}=740-N=600 \mathrm{tr} / \mathrm{mm}$.

7/ Développement des distorsions. $T=92-R e=1240-N=1004 \mathrm{tr} / \mathrm{mn}$

8) Apparition de la turbulence dans les tourbillons de Taylor. $T=183-R \mathrm{e}=2468-N=2000 \mathrm{tr} / \mathrm{mn}$

9/ Trace des tourbillons en écoulement essentiellement turbulent. $T=277-R \mathrm{e}=3730-N=3022 \mathrm{tr} / \mathrm{mn}$.

10/ Tourbillons de Taylor pour $C / R=0,0031$. $T=55-R \mathrm{e}=987-N=1514 \mathrm{tr} / \mathrm{mn}$.
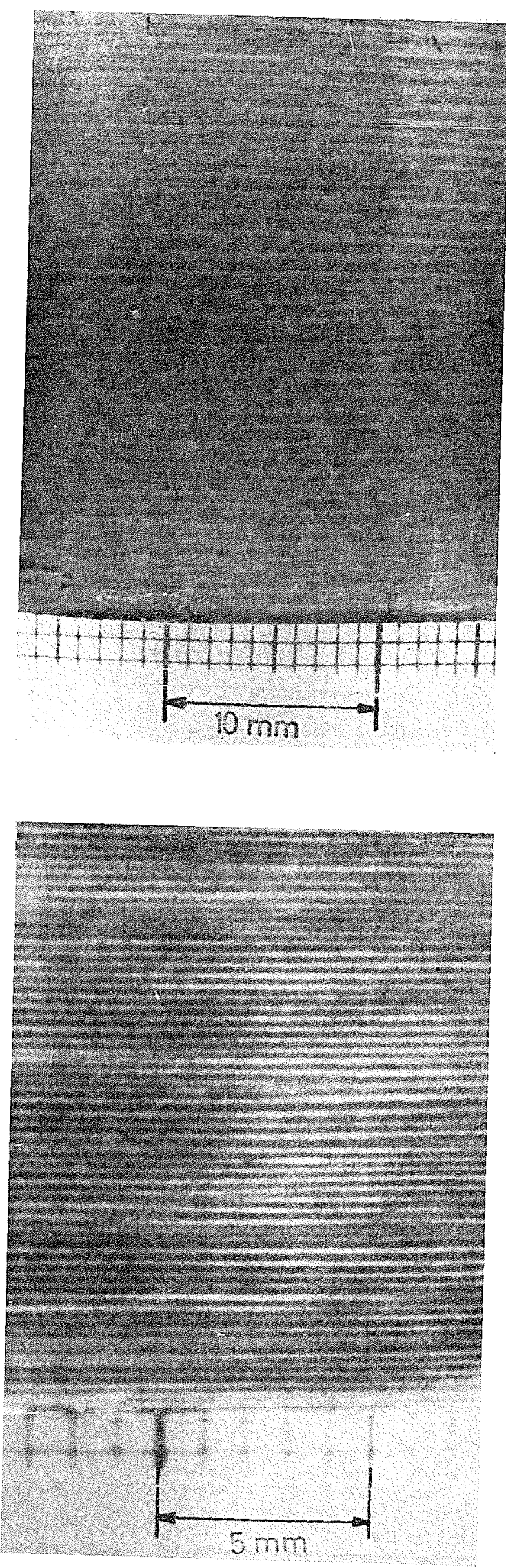


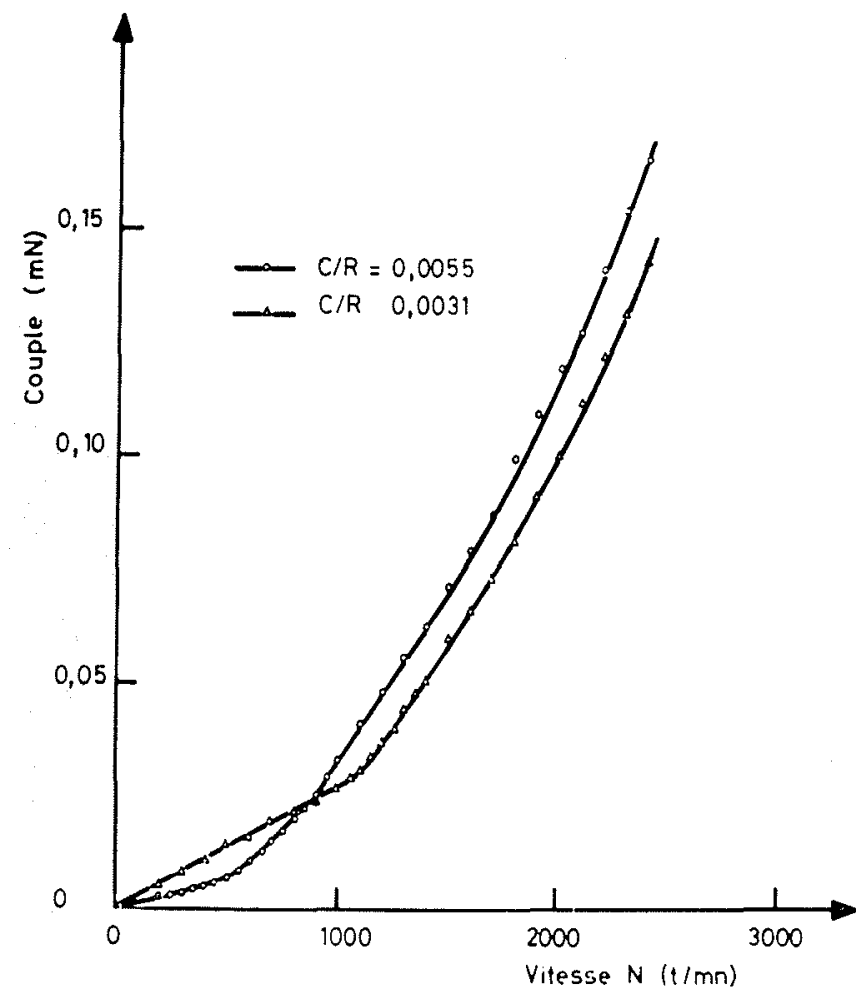

11/ Variation du couple avec la vitesse.

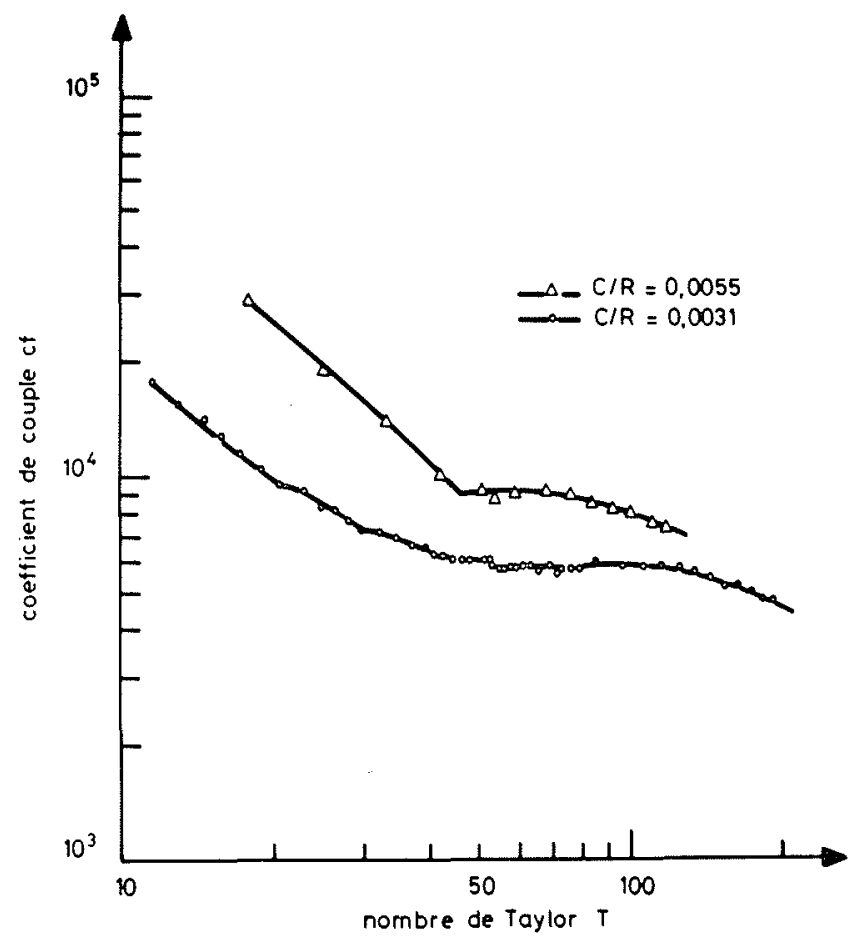

12/ Coefficient de couple en fonction du nombre de Taylor.

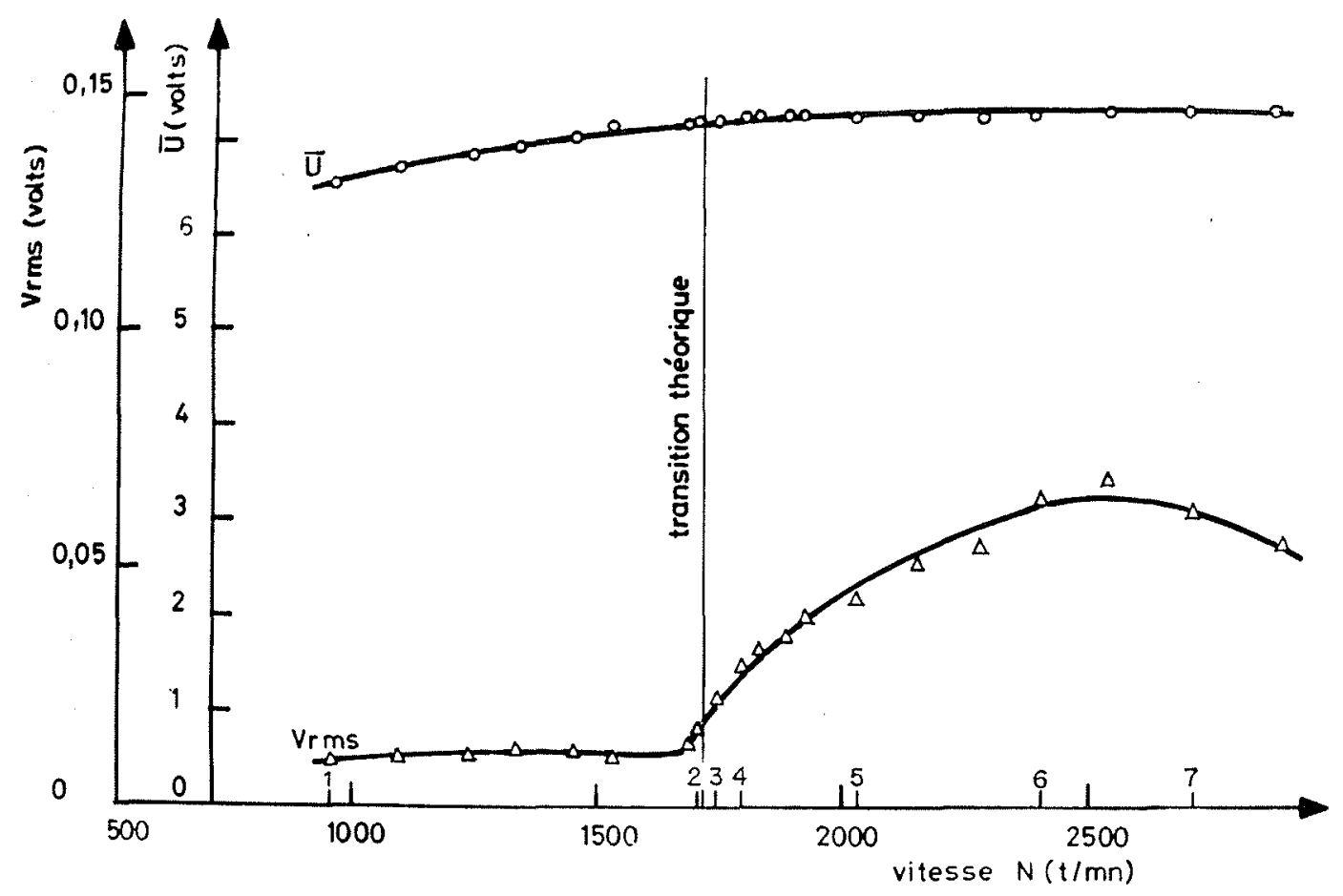

13/ Courbes de variation des tensions Vrms et $\bar{U}$ avec la vitesse dans le cas des cylindres coaxiaux. 

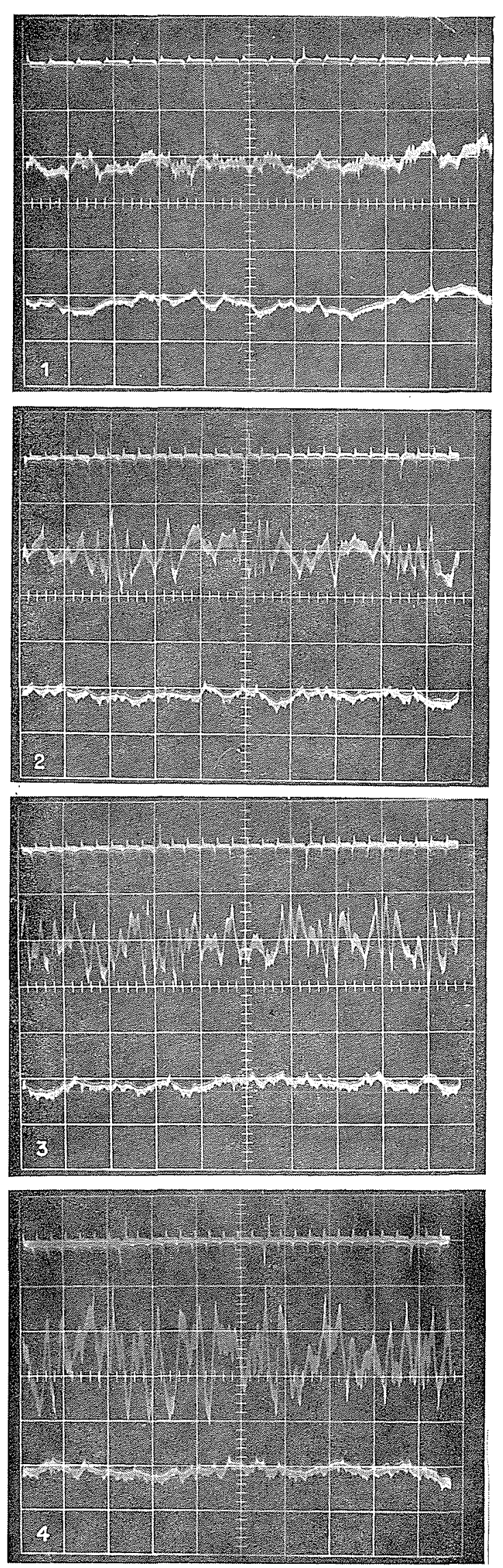
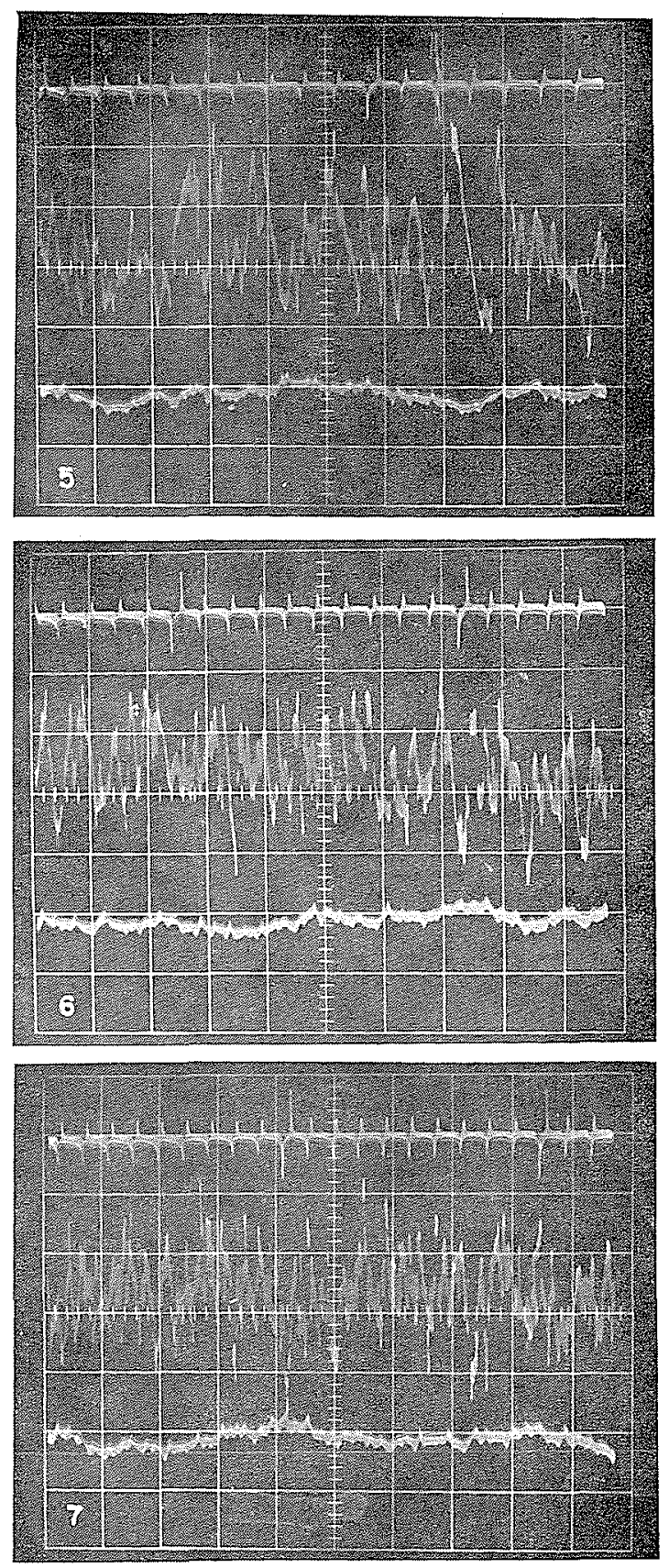

1. $N=955 \mathrm{trimn}-T=23-R e=469-$ tension $u^{\prime}=0,05 \mathrm{~V} / \mathrm{d}$ épaisseur $h=10 \mu \mathrm{m} / d$ - base de temps $=10 \mathrm{~ms} / d$.

2. $N=1702 \mathrm{ir} / \mathrm{mn}-T=41-R e=836-$ tension $u^{\prime}=0,05 \mathrm{~V} / d$ - epaisseur $h=10 \mu \mathrm{m} / d$ - base de temps $=10 \mathrm{~ms} / \mathrm{d}$.

3. $N=1744 \mathrm{tr} / \mathrm{mn}-T=42-R e=857-$ tension $u^{\prime}=0,05 \mathrm{~V} / \mathrm{d}$ - épaisseur $h=10 \mu \mathrm{m} / d$ - base de temps $=10 \mathrm{~ms} / d$.

4. $N=1796 \mathrm{tr} / \mathrm{mn}-T=43,2-R e=882-$ tension $u^{\prime}=0,05 \mathrm{~V} / \mathrm{d}$ - épaisseur $h=10 \mu \mathrm{m} / d$ - base de temps $=10 \mathrm{~ms} / d$.

5. $N=2024 \mathrm{tr} / \mathrm{mn}-T=48,7-R e=995-$ tension $u^{\prime}=0,05 \mathrm{~V} / \mathrm{d}$ - épaisseur $h=10 \mu \mathrm{m} / d$ - base de temps $=5 \mathrm{~ms} / d$.

6. $\mathrm{N}=2396 \mathrm{tr} / \mathrm{mn}-T=57,7-R e=1177-$ tension $u^{\prime}=0,05 \mathrm{~V} / \mathrm{d}$ - épaisseur $h=10 \mu \mathrm{m} / d$ - base de temps $=5 \mathrm{~ms} / \mathrm{d}$.

7. $N=2708 \mathrm{tr} / \mathrm{mn}-T=65,2-R e=1330-$ tension $u^{\prime}=0,1 \mathrm{~V} / \mathrm{d}$ - epaisseur $h=10 \mu \mathrm{m} / d$ - base de temps $=5 \mathrm{~ms} / d$.

14/ Photographies des signaux do l'oscilloscope dans le cas des cylindres coaxiaux. 


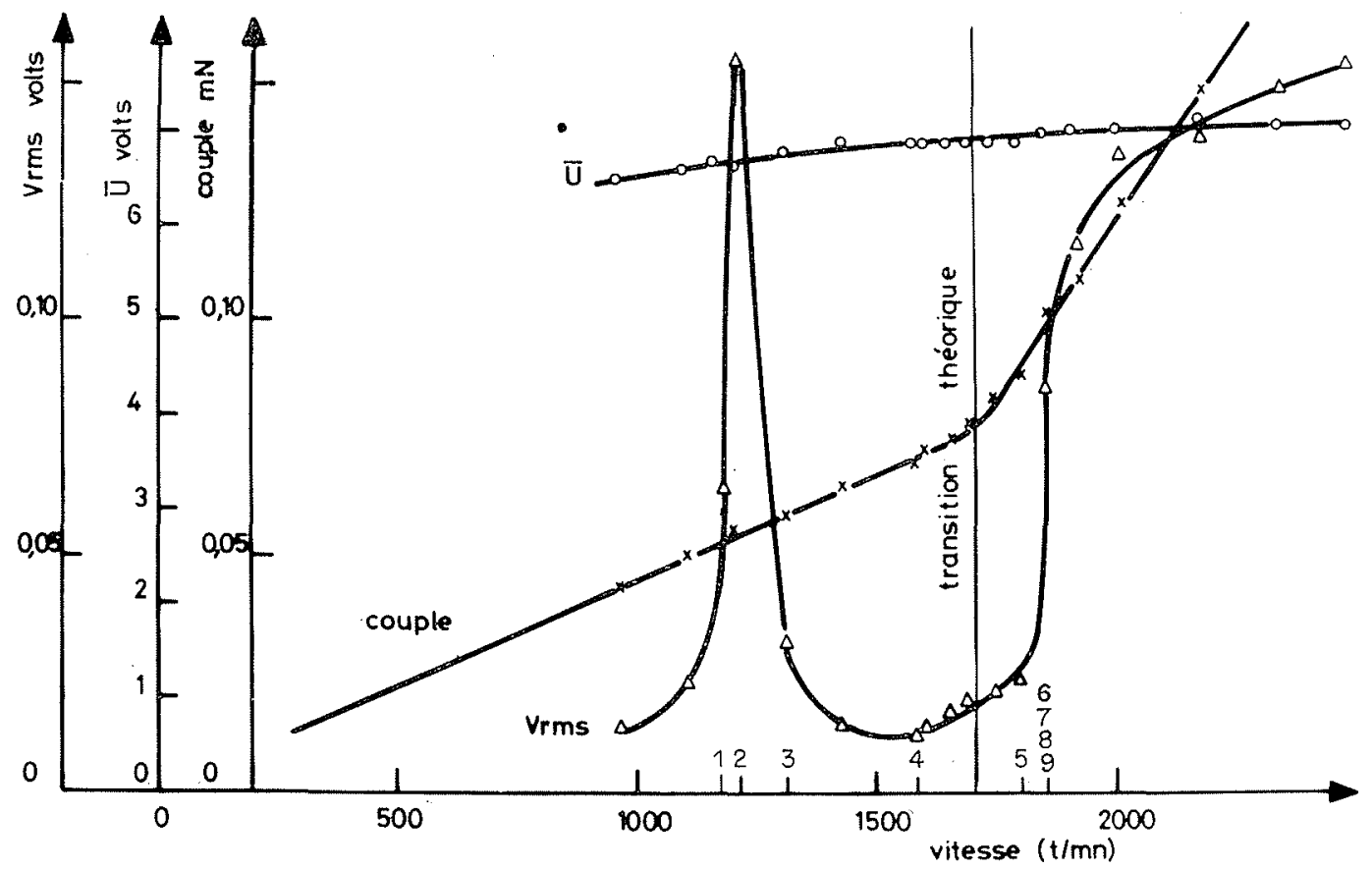

15/ Coubes de variation du couple et des tensions $V_{r i n}$ et $\bar{U}$ avec la vitesse en présence du fouettement
La figure 11 donne les variations du couple avec la vitesse pour les deux valeurs du jeu radial. A faible vitesse, les résultats expérimentaux sont en accord avec les valeurs calculées à nartir de la théorie laminaire. La cassure observée dans l'allure de ces courbes correspond à l'apparition des tourbillons de Taylor. Le couple augmente ensuite plus rapidement lorsque le jeu est plus important. Le coefficient de couple $C f$, défini par Schlichting [21] comme étant le rapport du couple mesuré $M_{0}$ sur le couple calculé en régime laminaire $M_{\mathrm{e}}$ que divise le quart du nombre de Reynolds $\mathcal{O}$ e, est présenté figure 12 en fonction du nombre de Taylor. Les changements de régime n'apparaissent malheureusement pas aussi clairement que dans le cas de jeux plus importants [21].

\section{Résultats obtenus par anémométrie dans le cas de cylindres coaxiaux}

Les essais ont été effectués pour une seule dimension du cylindre extérieur qui donne une jeu radial de $0,1 \mathrm{~mm}$, c'est-à-dire un rapport $C / R$ de 0,0024 . Au cours d'un essai, la température reste sensiblement constante et voisine de $26^{\circ} \mathrm{C}$; le maximum d'écart de température est de $1{ }^{\circ} \mathrm{C}$. La figure 13 donne les variations des tensions Vrms et $\bar{U}$ de la transition et les points, pour lesquels en fonction de la vitesse. La valeur théorique les photographies des signaux de l'oscilloscope ont été obtenues, sont portés sur cette figure. La figure 14 présente ces photographies dans les conditions suivantes :

14.1) en régime laminaire;

14.2) près de la transition;

14.3) juste après la transition;

$14,4,5,6$ et 7 ) au-delà de la transition.

Les signaux présentés sur ces photos correspondent, de haut en bas, à la vitesse angulaire en tours et en dixièmes de tours, à la tension $u^{\prime}$ de sortie de l'anémomètre et à l'épaisseur $h$ du film.

Les résultats obtenus par les techniques de visualisation ont montré que, pour deux cylindres coaxiaux, les tourbillons de Taylor apparaissent en accord avec la théorie même dans le cas de jeux très petits. La cassure notée (figure 13), vers $1700 \mathrm{tr} / \mathrm{mn}$, dans la courbe de variation de la tension Vrms avec la vitesse, est ainsi due à l'apparition dəs tourbillons de Taylor. Par ailleurs, les figures $14.1,2,3$, et 4 , prises respeotivement pour les vitesses de $955,1702,1744$ et $1796 \mathrm{tr} / \mathrm{mn}$, montrent très nettement la différence des signaux obtenus en régime laminaire et en régime de tourbillon de Taylor. Ainsi, la mesure de la contrainte de cisaillement à la paroi al moyen d'un anémomètre à film chaud permet, même dans le cas de jeu très réduit, de déterminer le point de transition au-delà duquel le régime n'est plus laminaire.
1.

$N=1179 \mathrm{tr} / \mathrm{mn}-T=28,4$

$\begin{aligned} R e & =579-T_{\min }=21,1 \\ T_{m 12} & =36,4-R\end{aligned}$

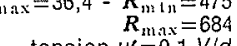

tension $u^{\prime}=0,1$ V/d

epe de iemps $=20 \mathrm{~ms} / \mathrm{d}$ excentricité relative $\varepsilon=0,18$

2.

$N=1205$ trimn $-T=29$ $R e=592-T_{1111}=13,5$

$T_{m a \mathrm{a}}=48,1-R_{m 1 \mathrm{~m}}=35 \mathrm{~s}$

tension $u^{\prime \prime \prime a x}=0.1$ V/O

aisseur $h=20 \mu \mathrm{m} / 0$

base de temps $=20 \mathrm{~ms} / 0$

3

$N=1310 \mathrm{tr} / \mathrm{mn}-T=31, \mathrm{~s}$ $N=1310 \mathrm{tr} / \mathrm{mn}-T=31, \mathrm{~L}$
$R e=644-T_{\mathrm{m} n \mathrm{n}}=2 \bar{t}$ $R e=644-T_{m \mathrm{nn}}=27$
$T_{\mathrm{max}}=36,4-R_{\mathrm{min}}=57 \mathrm{c}$
$R_{\mathrm{max}}=70 \varepsilon$ $\begin{aligned} & R_{\text {max }}=708 \\ & \text { tension } U^{\prime}=0,1 \mathrm{~V} / \mathrm{C}\end{aligned}$ épaisseur $h=40 \mu \mathrm{m} / \mathrm{c}$ base de temps $=20 \mathrm{~ms} / \mathrm{c}$ excentricité relative $\varepsilon=0,1$

$N=1583 \mathrm{tr} / \mathrm{mn}-T=38,1$ tension $u^{\prime}=0,1 / / c$ epaisseur $h=40 \mu \mathrm{m} / \mathrm{c}$ ase de temps $=20 \mathrm{~ms} / \mathrm{c}$ excentricité relative $\varepsilon=0,4$

$$
\begin{array}{r}
5 \\
N=1796 \mathrm{tr} / \mathrm{mn}-T=43, ; \\
R e=88, \\
\text { tension } u^{\prime}=0,05 \mathrm{~V} / \mathrm{C} \\
\text { epaisseur } h=20 \mu \mathrm{m} / \mathrm{c} \\
\text { base de temps }=10 \mathrm{~ms} / \mathrm{C}
\end{array}
$$

$N=1851 \mathrm{tr} / \mathrm{mn}-T=44$, $R e=910-T_{m+n}=22$; $T_{\max }=71,5-R_{\mathrm{min}}=57:$ tension $u^{\prime}=0,05 \mathrm{~V} / \mathrm{c}$ épaisseur $h=20 \mu \mathrm{m} / \mathrm{c}$ base de temps $=10 \mathrm{~ms} /$ excentricité relative $\varepsilon=0,3$

7

$N=1851 \mathrm{tr} / \mathrm{mn}-T=44$ $R e=910-T_{\text {min }}=22$, $\begin{aligned} T_{\mathrm{mix}} & =71,5-R_{\mathrm{min}}=57:\end{aligned}$ tension $u^{\prime}=01 \mathrm{~V}=1 /$ tension $h=0,1 \mathrm{k} /$

epaisseur $h=10 \mu \mathrm{m} /$ excentricité relative $\varepsilon=0,3$

$$
\begin{array}{r}
8 \\
N=1851 \mathrm{tr} / \mathrm{mn}-T=44, \\
R \mathrm{Re}=910-T_{\min }=22, \\
T_{m \mathrm{~m}}=71,5-R_{\min }=57 \\
R_{\max }=124 \\
\text { tension } U^{\prime}=0,1 \mathrm{~V} / \mathrm{c} \\
\text { epaisseur } h=40 \mu \mathrm{m} / \mathrm{c} \\
\text { base de temps }=5 \mathrm{~ms} / \mathrm{c} \\
\text { excentricité relative } \varepsilon=0,3
\end{array}
$$



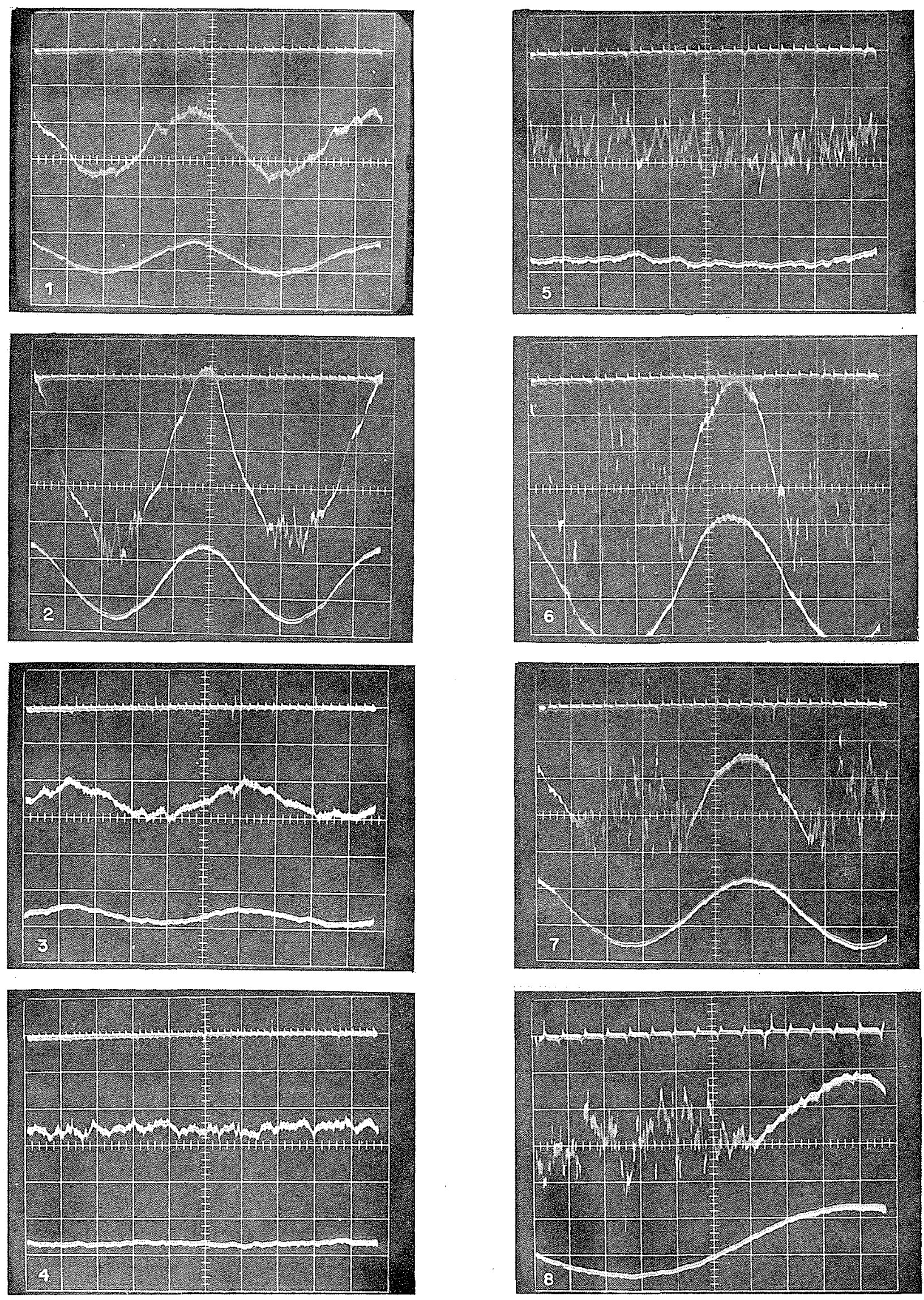

16/ Photographies des signaux de l'oscilloscope dans le cas des cylindres excentrés, sous l'action des instabilités mécaniques de fonettement. 
Résultats obtenus par anémométrie dans le cas de cylindres excentrés sous l'action de l'instabilité mécanique de fouetiement

Les essais ont été effectués avec le cylindre extérieur qui donne un rapport $C / R$ de 0,0024 . La figure 15 donne les variations du couple et des tensions Vrms et $\bar{U}$ dans le cas où le cylindre extérieur est libre de se déplacer radialement sous l'action des instabilités mécaniques de fouettement. La valeur théorique de la transition, calculée dans le cas des cylindres coaxiaux, ainsi que les points pour lesquels les photos ont été obtenues, sont portés sur cette figure. Les figures 16 présentent ces photographies prises dans les conditions suivantes:

16.1) avant la vitesse critique de fouettement;

16.2) à la vitesse correspondant à la fréquence de résonnance;

16.3) juste après la vitesse critiaue de fouettement;

16.4) avant la transition sans instabilité mécanique;

16.5) après la transition sans instabilité mécanique;

16.6, 7 et 8) en régime de demi-fréquence de fouettement.

La figure 15 montre l'effet des instabilités mécaniques de fouettement sur la tension Vrms qui ne caractérise plus l'existence des tourbillons de Taylor mais l'amplitude de la vibration. La cassure, dans la courbe de variation du couple avec la vitesse, montre que la transition s'effectue, en accord avec la théorie, en un point où les vibrations n'existent pas. Les figures $16.1,2$ et 3 montrent les variations des fluctuations $u^{\prime}$ et de l'épaisseur du film au droit de la sonde; les nombres de Taylor et de Reynolds, calculés pour les valeurs minimales, moyennes et maximales du jeu sont portés sur ces figures ainsi que l'excentricité relative $\varepsilon$. Le signal caractéristique des tourbillons de Taylor apparaît figure 16.2, alors que la valeur maximale du nombre de Taylor local est supérieur au nombre de Taylor critique calculé à partir des théories locales ou non locales. Lorsque la vitesse augmente, les instabilités mécaniques disparaissent (figure 16.4), le signal caractéristique des tourbillons de Taylor est observé dans tout le film (figure 16.5). Puis l'instabilité mécanique de fouettement réapparait (figures $16.6,7$, et 8 ). Sur ces trois dernières photos, on remarque que le signal caractéristique des tourbillons de Taylor est amplifié dans la zone de jeu maximum, pour disparaître et faire place au signal laminaire dans la zone de jeu minimum. Dans ce cas, la valeur minimale du nombre de Taylor est inférieure à la valeur critique, bien que celle du nombre de Taylor moyen soit supérieure à cette valeur critique.

\section{Conclusion}

Les résultats obtenus avec des jeux relatifs très petits $(C / R=0,0024,0,0031$ et 0,0055$)$ montrent que :

a) les tourbillons de Taylor existent même dans les espaces très petits, ils précèdent la turbulence;

b) la mesure du couple, qui permet de déterminer la transition entre le régime laminaire et le régime des tourbillons de Taylor, ne permet plus de caractériser la turbulence; c) la transition entre le régime laminaire et le régime des tourbillons de Taylor s'effectue brutalement, elle peut être caractérisée par un seul nombre qui dans le cas des cylindres coaxiaux est donné par la théorie; la transition entre le régime des tourbillons de Taylor et le régime turbulent s'effectue graduellement, elle ne peut donc pas être caractérisée par un seul nombre;

d) l'anémomètre à film chaud est capable de déterminer la transition entre le régime laminaire et le régime des tourbillons de Taylor;

e) dans le cas des cylindres excentrés les tourbillons apparaissent dans la zone de jeu maximal dès que le nombre de Taylor local dépasse la valeur du nombre de Taylor critique, même si le nombre de Taylor moyen est plus petit que cette valeur critique.

Ces derniers résultats sont plus en accord avec les théories basées sur le concept de stabilité locale, que celle qui tient compte d'une vitesse radiale dans le fluide. Il faut cependant noter que ces résultats ont été obtenus en fonctionnement dynamique, ce qui n'est pas exactement les conditions décrites dans les théories.

\section{Remerciements}

Ce travail a été effectué grâce au financement de l'Electricité de France, Direction des Etudes et Recherches, Service Machines et Automatismes de production, à Chatou, contrat $\mathrm{n}^{\circ} \mathrm{HJ} / 033 / 14.173$.

\section{Bibliographie}

[1] TAYloR (G. I.). - Stability of viscous liquid contained between two rotating cylinders. Philosophical Transactions, série A vol. 223 (1923), p. 289-343.

[2] FrênE (J.). - «Contribution à l'étude des régimes de transition dans les paliers lisses ». Thèse présentée à la Faculté des Sciences de l'Université de Lyon (17 octobre 1970).

[3] Di Prima (R.C.). - A note on the stability of flow in loaded journal bearing. Transactions of the American Society of Lubrication Engineers, vol. 6, $\mathrm{n}^{\circ} 3$ (1963), p. 249-253.

[4] Ritchie (G.S.). - On the stability of viscous flow between eccentric rotating cylinders. Joumal of Fluid Mechanics, vol. 32, part 1 (1968), p. 131-144.

[5] Urban (R. L.) et Krueger (E. R.). - On the stability of viscous flow between two rotating non-concentric cylinders. Joumal of the Franklin Institute, vol. 293, n' 3 (1972) p. 153-172.

[6] Di Prima (R. C.) and Stuart (J. T.). - Non-local effects in the stability of flow between eccentric rotating cylinders. Journal of Fluid Mechanics, vol. 54, part 3 (1972), p. 393-415.

[7] Castle (P.) et Monss (F. R.). - Hydrodynamic stability of the flow between eccentric rotating cylinders: visual observation. and torque measurements. Proceeding of the Institution of Mechanical Engineers, vol. 182, part $3 \mathrm{~N}$ (1967-1968), p. 41-52.

[8] Younes (M. A.), Mobis (F. R.) et Coney (J. E.). - Hydrodynamic stability of the flow between eccentric rotating cylinders with axial flow: torque measurements. Proceeding of the Institution of Mechanical Engineers. Tribology Convention (1972), C 76/72, p. 14-19. 
[9] FRÊNE (J.) et Goder (M.). - La portance et le couple résistant d'un palier lisse fonctionnant en régime non laminaire. Mécanique, Matériaux, Electricité GAMI, $\mathrm{n}^{\circ} 274$ (octobre 1972), p. $18-29$.

[10] FrêNe (J.) and Godet (M.). - Transition from laminar to Taylor vortex flow in journal bearing. Tribology, vol. 4, $\mathrm{n}^{\circ} 4$ (1971), p. 216-217.

[11] Cole (J. A.). - Experiments of Taylor vortices between eccentric rotating cylinders. The Second Australasian Conference on Hydraulics and Fluid Mechanics, (1965), p. B 313-B 320.

[12] Cole (J. A.). - Taylor vortices with eccentric rotating cylinders. Nature, vol. 221 (1969), p. 253-254.

[13] Kamal (M. M.). - Separation in the flow between eccentric rotating cylinders. Transaction of the American Society of Mechanical Engineers, Journal of Basic Engineering, série D, vol. $88, \mathrm{n}^{0} 4$ (1966), p. 717-724.

[14] VoHR (J.H.). - An experimental study of Taylor vortices and turbulence in flow between eccentric rotating cylinders. Transaction of the American Society of Mechanical Engineers, Journal of Lubrication Technology, série F, vol. 90, $\mathrm{n}^{\circ} 1$ (1968), p. 285-296.

[15] Castle (J.), Mobbs (F. R.) et Markho (P. H.). - Visual observations and torque measurements in the Taylor vortex regime between eccentric rotating cylinders. Transaction of the American Society of Mechanical Engineers, Journal of Lubrification Technology, série F, vol. 93, no 1 (1971), p. 121-129.

[16] Bowman (R. M.), Collingwood (J. C.) et Midgley (J. W.). Some factors affecting the whirl instability of a journal bearing: Part 1. Proceeding of the Institution of Mechanical Engineers. Lubrication and Wear Convention (23rd-26th May 1963), p. 18-26.

[17] FrêNE (J.) and Godet (M.). - Flow transition criteria in a journal bearing, presented at the A.S.M.E., Spring Lubrication meeting (June 4th-6th 1973). Northwestern University, Evanston, U.S.A., paper $\mathrm{n}^{\circ} 73$, Lub $\mathrm{S} 13$.

[18] FRÊNE (J.) et GODET (M.). - Détermination par sonde pariétale du changement de régime de l'écoulement entre deux cylindres excentrés de rayon très voisin. C.R. Acad. Sc. Paris, t. 276 série A (16 avril 1973), p. 1133-1136.

[19] FRÊNE (J.) et GODET (M.). - Detection of Taylor vortex transition in very small clearances by hot film wall anemometry. Tribology, vol. 6, no 5 (October 1973), p. 178-183.

[20] Cores (D.). - Transition in circular Couette flow. Joumal of Fluid Mechanics, vol. 21, part 3 (1965), p. 385-425.

[21] Schlichting (H.). - Boundary Layer theory. Me Graw Hill series, in Mechanical Engineering, 6th edition (1968), p. 500-503. 